\section{Blood Vomiting after Work on Spray Paint}

\author{
Zhong Jia*, Chao He", Liang-Liang Fang" and Yue Zhou \\ Hangzhou First People's Hospital, Nanjing Medical University Affiliated \\ Hangzhou Hospital \& Zhejiang Chinese Mediciene Affiliated $4^{\text {th }}$ Clinical \\ Teaching Hospital, China \\ \# First Authors
}

paint, when compared to her previous medical health record. Hence, she highly suspected that her symptoms might be associated with her work environment or some paint solvents. She and her family hoped clinicians could confirm or exclude her suspicion during therapeutic course in this admission. Her words arouse our great interest and we decided to decode the conundrum. Amazingly, no specific clues were obtained from her family history, appetites and general laboratory tests. Further enhanced contrast computed tomography of abdomen showed special findings, including isolated gastric varices, major portal vein thrombosis (red arrowhead), focal splenic vein stenosis (blue arrowhead), and splenomegalia complied with focal splenic, but myelofibrosis was confirmed by bone marrow biopsy and the expression of JAK2-V617F was detected in her peripheral blood sample (Figure 1).

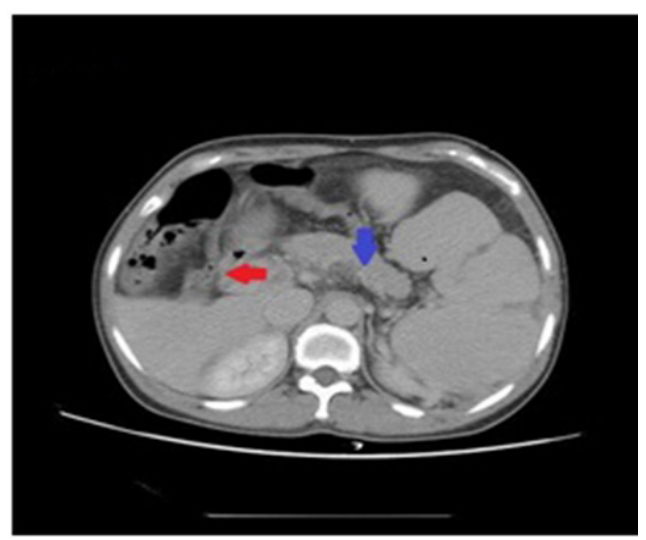

Figure 1: Special findings, including isolated gastric varices, major porta vein thrombosis (red arrowhead), focal splenic vein stenosis (blue arrowhead) and splenomegaly complicated with focal splenic.

According to hematologists advices, patients with positive JAK2V617F gene are usually not suitable for splenectomy, because this gene is closely associated with diagnosis of some blood diseases such as myelofibrosis in this case $[5,6]$. What physicians fear is the fact that uncontrollable elevation of peripheral blood cells, and its potential disaster secondary to these changes such as thrombocytosis, while surgeons insisted on performing splenectomy plus devascularization of gastric varices as the patient was diagnosed as left-sided portal hypertension based on CT findings, if not so, the potential of rebleeding from varices wouldn't be resolved $[7,8]$, which might push the patient into risk of life-threatening.

\section{Presence and Strategy}

Left-sided portal hypertension is a rare disease mainly resulting from splenic vein occlusion in various possible ways $[7,8]$. According to current studies $[7,8]$, pancreatogenous disorders are the most common causes of splenic vein obstruction, while extra-pancreatogenous etiologies, including idiopathic cause, blood disease and splenic vein lesion itself. And splenic vein stenosis may has some relation with the idiopathic cause [9]. In this case, the splenic vein stenosis might be indeed associated with positive JAK2-V617F gene and related myelofibrosis rather than idiopathic etiology. 
Patients with positive JAk2-V617F can produce some cytokines, which may lead to vessel and epithelia endothelial cells proliferation and its fibrosis formation. This may be a definitive reason why splenic vein stenosis disease in this case was ultimately formed. And then, both thrombocytosis and splenic vein stenosis resulted in portal vein thrombosis, while these changes may account for congestive splenomegaly and gastric varices. The differential diagnosis includes blood diseases, post-cirrhotic portal hypertension and Budd-Chiari syndrome, etc.

Most of symptomatic patients with left sided portal hypertension can be treated with splenectomy alone or plus devascularization operation, the minority of patients may choose conservative therapy. In this case, we have to face another layer of complex with two different risks. At the first layer, conservative therapy may be the priority of choice and endoscopic technique can be applied to stop bleeding due to occasional gastric varices rupture. But on second look, how to resolve early satiety and abdominal distention triggered by gradually enlarged spleen?

As a matter of fact, sometimes it's necessary to take adventure if benefits from splenectomy beyond the potential risks of myelofibrosis. For example, we have ever encountered a gastric body cancer patient with myelofibrosis, who must receive total gastroectomy plus splenectomy. Based on our previous similar experience, the crucial key to success is better administration of myelofibrosis after surgery than fear.

\section{Problem Resolving}

In this case, laparoscopic splenectomy plus devascularization and wedge liver biopsy were performed successfully; figure 2 aiming to improve the patient's quality of life and to reduce the risk of rebleeding of gastric varices.

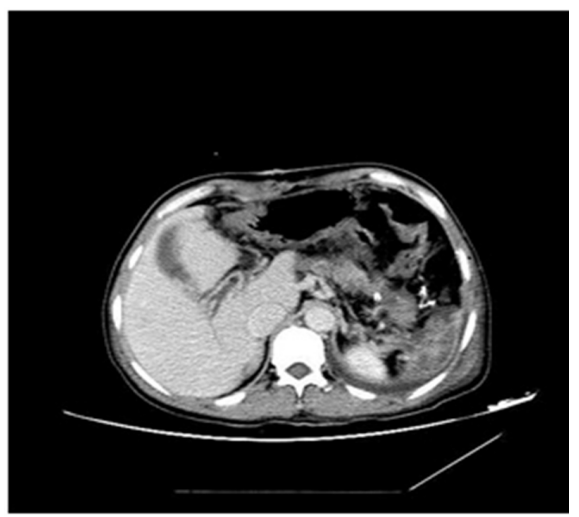

Figure 2: Laparoscopic splenectomy plus devascularization and wadge liver biopsy were performed successfully.

The histopathological assessment confirmed the non-cirrhotic liver (Figure 3 ) and congestive splenomegaly combined with focal splenic infarction (Figure 4). Obviously, the pathologic results kept coincide with initial diagnosis of left-sided portal hypertension.

Although antiplatelet therapy (aspirin intake add venous heparin) was conducted to treat portal vein thrombosis, the platelet count seemed to be out of control, with sharply rising and up to its peak $(1,620,000 / \mu \mathrm{L}(125000 / \mu \mathrm{l}-350000 / \mu \mathrm{l}))$ at postoperative day 21 . In fact, the backlash of platelet number was just a normal response to splenectomy, while impact of myelofibrosis was minor. Whatever it moved, enhancing antiplatelet therapy/hydroxyurea intake adding interferon) was administrated. As usual, heparin and warfarin underwent overlapping use for 5 days before discharge. Fortunately, thanks to positive response to therapies, the platelet count began to decline at postoperative day 22 without any signs of rebound to rising. After discharge, the patient was administrated by oral warfarin intake alone. In review, 3 months after surgery, she recovered with normal platelet count and without evidence of rebleeding. But a few months later, we received the call that when the patients stop taking antiplatelet medicine the platelet count will fluctuate may be result of the splenectomy or withdrawal symptoms.

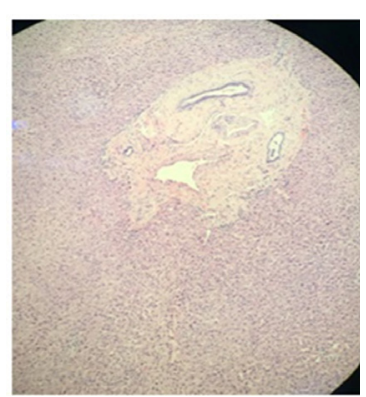

Figure 3: A histopathological assessment confirmed the non-cirrhotic liver.

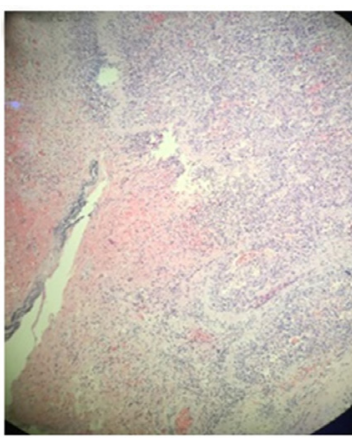

Figure 4: Congestive splenomegaly combined with focal splenic infarction.

\section{Discussion}

JAK2-V617F genes are still unable to find a particular incentive. Radiation, certain contrast agents and some industrial solvents (such as benzene, toluene) may increase the risk of disease [1-4]. In fact, benzene and toluene are usually used as paint solvents. In this case, long-term working on spray paint was very dangerous as she had no safeguard procedures to avoid contacting these industrial solvents. The patient's suspicion may be likely real. Splenic vein stenosis might be indeed associated with positive JAK2-V617F gene and related myelofibrosis. Studies have shown that myeloid cells are differentiated from clonal stem cells in patients with primary myelofibrosis. However, bone marrow fibroblasts and $\mathrm{T}$ cells are polyclonal. The cause of excessive myelofibrosis is still unknown. Platelets, megakaryocytes and mononuclear cells is secreting cytokines, such as Transforming Growth Factor beta (TGF beta), Platelet-Derived Growth Factor (PDGF), Interleukin-1 (IL-1), Epidermal Growth Factor (EGF) and basic Fibroblast Growth Factor (bFGF), 3 which can cause fibroblast 
growth and proliferation of extracellular matrix. In addition, TGF beta and bFGF beta may cause proliferation of vascular endothelial cells and growth of bone marrow micro vessels. Consequently, the splenic vein stenosis may be accessible, while portal thrombosis and splenomegaly may be accordingly emerged.

This may be a likely reason why splenic vein stenosis disease in this case was ultimately formed. And then, both thrombocytosis and splenic vein stenosis resulted in portal vein thrombosis, while these changes may account for congestive splenomegaly and gastric varices. And the ruptures of gastric varcies cause the blood vomit of this 51 years old female spray paint worker.

Meanwhile, up to date, the overwhelming majority of the patients with positive. The incidence of secondary hematologic and non hematologic malignancies was increased in patients with chronic myeloid proliferative tumors. Compared with the incidence rate of the general population, the risk of having two tumors in patients with primary thrombocytosis is 1.2 times; the patients with polycythemia vera are 1.6 times; the patients with chronic myelogenous leukemia are 1.6 times.

Interestingly, hypersplenism counteracted effects of thrombosis before surgery; as a result, the platelet count seemed to be within normal range before surgery. But elevation of platelet may be temporarily a natural response to splenectomy. On the other hand, the elevation of platelet after splenectomy also may be another positive echo to curb the progress of myelofibrosis, but interconnected ties between them still remains unclear and waits for further study.

\section{Summary}

The unique but instructive case will be worthy of reading, aiming to appeal the whole society to pay more attention on people's working environment to enhance safeguard steps. And when we face symptomatic left-sided portal hypertension associated with JAK2-V617F, splenectomy as a major therapy is safe and effective if antiplatelet therapy after surgery is better administrated.

\section{Informed Consent and Ethical Approval}

The case report has obtained informed consent from the patient and her family. Also, we have got the approval of ethic committee in our institution.

\section{Declaration}

All authors declare that they have no conflict interests concerning on the manuscript.

\section{References}

1. Reilly JT (1997) Idiopathic myelofibrosis: pathogenesis, natural history and management. Blood Reviews 11: 233-242.

2. Bachleitner-Hofmann T, Gisslinger H (1999) The role of interferon- $\alpha$ in the treatment of idiopathic myelofibrosis. Annals of hematology 78: 533538.

3. Yogarajah M, Tefferi A (2017) Leukemic Transformation in Myeloproliferative Neoplasms: A Literature Review on Risk, Characteristics, and Outcome. Mayo Clin Proc 92: 1118-1128.

4. Cervantes F, Dupriez B, Pereira A, Passamonti F, Reilly JT, et al. (2009) New prognostic scoring system for primary myelofibrosis based on a study of the International Working Group for Myelofibrosis Research and Treatment. Blood 113: 2895-2901.

5. Foucar CE, Stein BL (2017) JAK2 V617F Mutation Testing in Patients Presenting With Hepatic and Portal Vein Thrombosis. JAMA 317: 22282229.

6. McKerrell T, Park N, Chi J, Collord G, Moreno T, et al. (2017) JAK2 V617F hematopoietic clones are present several years prior to MPN diagnosis and follow different expansion kinetics. Blood Adv 1: 968-971.

7. Hakim S, Bortman J, Orosey M, Cappell MS (2017) Case report and systematic literature review of a novel etiology of sinistral portal hypertension presenting with UGI bleeding: Left gastric artery pseudoaneurysm compressing the splenic vein treated by embolization of the pseudoaneurysm. Medicine (Baltimore) 96: 6413.

8. Ergenc H, Eminler A T, Varim C, Karacaer C, Uslan Mİ, et al. (2015) Gastric variceal bleeding related with sinistral portal hypertension occurring secondary to splenic vein thrombosis developed as a complication of acute pancreatitis. Int J Adv Med 2: 419-422.

9. Wong HK, Kuo HT, Tsai SL, Uen YH, Tzeng WS (2004) Isolated gastric varices due to focal splenic vein stenosis. J Formos Med Assoc 103: 640643. 